\title{
Metastatic gastric cancer to the female genital tract (Review)
}

\author{
HIROSHI MATSUSHITA, KAZUSHI WATANABE and AKIHIKO WAKATSUKI
}

Department of Obstetrics and Gynecology, Aichi Medical University School of Medicine, Nagakute, Aichi 480-1195, Japan

Received May 9, 2016; Accepted July 7, 2016

DOI: $10.3892 /$ mco.2016.1035

\begin{abstract}
Metastases to the female genital tract from gastric cancer are rare, but they significantly worsen the prognosis of such patients. The potential routes for metastasis to the female genital tract from gastric cancer include hematogenous spread, lymphatic spread and surface implantation. The rate of lymphatic metastasis to the ovary from gastric cancer has been reported to be higher compared with that from colorectal cancer. Uterine or Fallopian tube metastases are usually secondary to ovarian metastases, which are typically identified prior to the detection of gastric cancer in half of all synchronous cases, with complaints of abdominal distention, pain, palpable mass, or abnormal uterine bleeding. The prognosis of patients with female genital tract metastases from gastric cancer is extremely poor, and is worse compared with that of other primary sites, such as the breast and colorectum. In the past, surgical intervention in such patients consisted mainly of palliative resection to relieve the symptoms associated with a sizeable pelvic mass. However, recent retrospective studies based on a relatively small number of patients have reported that surgical tumor debulking plus chemotherapy may improve the prognosis of patients with metastatic ovarian cancer originating from gastric cancer.
\end{abstract}

\section{Contents}

1. Introduction

2. Characteristics

3. Clinical presentations

4. Diagnosis

5. Management and prognosis

6. Conclusions

Correspondence to: Dr Hiroshi Matsushita, Department of Obstetrics and Gynecology, Aichi Medical University School of Medicine, 1-1 Yazakokarimata, Nagakute, Aichi 480-1195, Japan E-mail: hirosm@aichi-med-u.ac.jp

Key words: gastric cancer, Krukenberg tumor, metastasis, ovary, surgery, uterus

\section{Introduction}

Gastric cancer, is the fourth most common type of cancer and the second leading cause of cancerrelated mortality, with $>$ 400,000 newly diagnosed cases annually worldwide (1). Although metastases to the female genital tract from gastric cancer are rare, women with such metastases are diagnosed with stage IV disease. The prognosis of patients with female genital tract metastasis from gastric cancer is extremely poor, and worse compared with that from other primary sites, such as the breast and colorectum.

The aim of this study was to review the relevant literature and highlight the characteristics, symptoms, diagnosis, management and prognosis of gastric cancer metastatic to the female genital tract.

\section{Characteristics}

Ovary. Metastasis to the female genital tract from extragenital neoplasms is rare. Mazur et al (2) analyzed 325 cases of metastasis to the female genital tract and reported that only $149(45.8 \%)$ were of extragenital primary origin, with the ovaries being the most frequently affected site $(75.8 \%)$, regardless of the location of the primary tumor. The authors (2) also reported that the most common sites of the primary tumor were the breast and the colorectum, followed by the stomach. This distribution of primary sites has been supported by reports from Western countries (3-6). By contrast, reports from Asia have emphasized that the stomach is the most common primary site (7-9), suggesting that the relative frequencies of various primary tumors are considered to simply reflect the prevalence of these tumors in the population at risk (2). However, gastric cancer has been reported to be the leading primary site in Krukenberg tumor, which is defined as a gastrointestinal cancer that has metastasized to the ovaries and contains a significant component of mucin-filled signetring cells typically lying within a cellular stroma derived from ovarian stroma $(10,11)$, regardless of the region $(3,8,9,12-15)$.

Although it is not fully understood why gastric cancer is more common than colorectal cancer as the primary cause of Krukenberg tumors, the differences in the metastatic pathways from the primary site may be partially explanatory. The potential routes for gastric cancer metastasis to the ovary include hematogenous spread, lymphatic spread and surface implantation (11). Yamanishi et al (11) examined the microscopic findings from metastatic ovarian cancers and found that the rate of lymphatic metastasis to the ovary from the stomach 
was significantly higher compared with that from the colon. By contrast, the authors also indicated that the rate of intravascular metastasis to the ovary from the colorectum tended to be higher compared with that from the stomach. Kim et al (16) reported that the incidence of ovarian metastasis from gastric cancer was closely associated with the extent of lymph node metastasis using a multifactorial analysis of 690 female gastric cancer patients. Recently, Feng et al (17) demonstrated that gastric cancer patients with $>6$ lymph nodes were more likely to have ovarian metastasis.

In addition, ovarian metastases from gastric cancers tend to be Krukenberg tumors, with the characteristics of being bilateral rather than unilateral $(3,4,6,7,14,17-20)$ and predominantly solid $(3,21)$. Furthermore, it has also been reported that ovarian metastases from gastric cancer are more commonly found in premenopausal rather than in postmenopausal women $(17,19,22,23)$. Miller et al (24) hypothesized that the higher blood flow in premenopausal ovaries may increase the risk of metastasis to the ovary.

Fallopian tube. Metastasis from gastric cancer involving only the Fallopian tube is extremely rare, with only 1 case, which was incidentally identifed, reported to date (25). Metastases to the Fallopian tube from non-gynecological sites are commonly found to be concurrent with ovarian metastases $(10,26,27)$. Kiyokawa et al (10) found 32 metastases to the Fallopian tube among 120 cases $(26.7 \%)$ of Krukenberg tumors of the ovary.

The mechanisms underlying Fallopian tube metastases remain unknown. However, all 3 potential routes for ovarian metastases, including lymphatic, vascular and peritoneal spread, are likely to be involved, with the lymphatic pathway considered to be the most common route (25). Stewart et al (26) examined 31 patients with ovarian metastases from non-gynecological origins and found Fallopian tube involvement in 20 patients. All 6 patients with ovarian metastases from gastric or presumptive gastric cancer were documented to have Fallopian tube metastases. Of note, metastatic cancer in all 6 cases showed signet ring cell morphology and intravascular involvement; in some cases, the metastatic tumor to the Fallopian tube also exhibited intraluminal involvement $(26,27)$. In addition to the 3 abovementioned potential routes, this finding may suggest an active process by the Fallopian tube suctioning tumor cells, possibly resulting in metastases to the Fallopian tube and the uterus (25).

Uterus. The uterus, including the corpus and the cervix, is also a rare metastatic site in extragenital cancers, with $\sim 200$ cases reported to date (28-30). Piura et al (28) reported that breast cancer was the most common primary site (56.6\%) and gastric cancer was the second, accounting for $16.7 \%$ of primary cancers.

The mechanisms of metastasis to the uterine corpus from gastric cancer are not clearly understood. Kumar and Hart (31) examined the anatomic distribution of non-gynecological cancer metastases within the uterine corpus. The metastases were almost always found in the myometrium, with or without endometrial involvement (96.2\% of cases), suggesting that lymphatic and hematological spread, as well as direct intraluminal spread from the ovary through the Fallopian tube, represent possible underlying mechanisms. The authors also investigated the distribution of other metastases in autopsy cases with metastases to the uterine corpus from extragenital cancers, and found that all cases were positive for lymph node metastasis but lacked an ovarian metastasis in $35.0 \%$ of the cases (31). The majority of metastases to the uterine corpus are considered to be secondary to local lymphatic spread from a preceding ovarian metastasis, while spread is likely hematological when the ovaries are not affected $(28,29,31,32)$.

The uterine cervix is less frequently affected compared with the uterine corpus, comprising only $20 \%$ of total uterine metastases (28). The potential routes for metastasis of non-gynecological carcinomas to the uterine cervix include hematogenous spread, retrograde lymphatic spread and transperitoneal seeding, with either transtubal spread or penetration from the cul-de-sac (33). The reasons for the relative rarity of metastases to the uterine cervix have not been fully elucidated, but the high fibromuscular tissue content and low degree of vascularity of the cervix are considered to be an unfavorable medium for metastatic growth (33). In addition, the cervix has lymphatic channels that drain centrifugally, and metastasis via this route occurs only when distant channels are blocked by the tumor and do not allow for retrograde flow (34).

Vagina. The vagina is the second most frequent metastatic site after the ovary (2). Primary adenocarcinoma of the vagina is extremely rare, accounting for $9 \%$ of all vaginal tumors. Adenocarcinoma of the vagina should be considered metastatic until proven otherwise. Approximately $65 \%$ of metastatic adenocarcinomas of the vagina develop from the cervix, endometrium, colon or ovary. However, metastasis of gastric cancer to the vagina is even less common $(2,35,36)$. A remote metastasis to the vagina from gastric cancer has not been reported in the literature to date, suggesting that metastases to the vagina from gastric cancer are likely extensions from the metastases to the ovary or uterus (37). It has not been fully elucidated why the vagina is rarely a remote metastatic site of gastric cancer. Vaginal metastases are considered to be most likely due to a venous pathway of spread through the vertebral venous system (a system that is constantly subjected to arrests and reversals in the direction of blood flow). Cancer cells from the stomach are considered to spread to the ovarian plexus or to the parametrial veins, and then to the vaginal vein (38). By contrast, lymphatic spread is the main pathway for metastases to the ovary and uterus from gastric cancer $(11,33)$.

Vulva. Metastatic tumors involving the vulva are extremely rare, with only a few case reports and series reported in the literature. Non-gynecological primary tumors may also metastasize to the vulva, accounting for $18.2-43.9 \%$ of cases $(39,40)$. However, to the best of our knowledge, no cases of metastasis to the vulva from gastric cancer have been reported to date $(2,39,40)$.

\section{Clinical presentations}

Metastasis to the ovary is often identified prior to the detection of the primary tumor, as the metastasis becomes symptomatic before the primary tumor (14). Kim et al (20) analyzed 73 women with ovarian metastases from gastric cancer and reported that 29 cases $(39.7 \%)$ were synchronous and 
44 cases $(60.3 \%)$ were metachronous, with a median time to diagnosis of 15.5 months (range, 5-46 months). In synchronous cases, ovarian metastases are detected prior to the primary tumor in $40.9-58.5 \%$ of the cases $(5,14)$. Abdominal distention, pain or a palpable mass is the most usual clinical presentation in patients with ovarian metastases $(3,6,19,20,23)$. It is noteworthy that abnormal uterine bleeding is not a rare presentation, even in postmenopausal women $(6,19)$. Rarely, patients may present with abnormal uterine bleeding from endometrial hyperplasia or virilization due to the excess estrogens produced by the luteinized stroma in a malignant ovary $(41,42)$.

Abnormal uterine bleeding is often the first presentation in women with uterine metastasis, with or without a history of malignancy $(28,29,31)$. In a series of 52 non-gynecological cancer metastases within the uterine corpus reported by Kumar and Hart (31), the authors found that the endometrium was involved in only $36.5 \%$ of the cases. In these cases, however, the patients could be asymptomatic, without uterine bleeding (28). Genital bleeding is also the most frequent symptom, followed by ascites and lower abdominal pain, in women with metastasis from gastric cancer to the uterine cervix (43). Rarely, metastasis to the uterine cervix from gastric cancer presents with ureteral obstruction $(30,44)$, although it is extremely rare for these lesions to be found at the metastatic site mimicking advancedstage disease (International Federation of Gynecology and Obstetrics stage IIIB).

\section{Diagnosis}

The ovary is the most frequent site of metastatic tumors (2). It has been reported that the incidence of all cancers (gynecological and non-gynecological) and non-gynecological metastatic ovarian cancer accounts for $8.8-27.8 \%(3,4,6,8,18,45)$ and $2.31-1.3 \%$ of all ovarian malignancies $(3-6,8,14,18)$, respectively. Distinguishing between primary and metastatic tumors, particularly non-gynecological, is crucial, as misinterpretation of a metastatic tumor as a primary tumor may lead to inappropriate management and suboptimal treatment outcomes $(5,6)$.

In cases when the patients do not have or fail to provide information regarding a history of malignancy, this may pose a major diagnostic challenge to the gynecologist and pathologist. Therefore, physicians should inquire whether patients have a history of gastric cancer. In women with pelvic masses, preoperative assessments include imaging studies and the evaluation of tumor markers to distinguish metastatic ovarian tumors from primary ovarian cancers. In patients without a prior history of gastric cancer, gastric and colon fiberscopy are also recommended. Imaging findings of metastasis in the ovary may be non-specific, but Krukenberg tumors display some distinctive characteristics, including bilateral complex masses with hypointense solid components (dense stromal reaction) and internal hyperintensity (mucin) on T1- and T2-weighted magnetic resonance imaging (MRI) $(41,46)$. Signal voids are frequently observed within the tumor, representing increased tumor vascularity, while solid components are usually enhanced homogeneously on contrast-enhanced computed tomography and T1-weighted MR images (41).

Serum cancer antigen (CA) 125 is a novel serum biomarker used for the management and diagnosis of primary epithelial ovarian cancer. However, its utility for metastatic tumors has not been fully elucidated. Turan et al (9) reported that CA125 levels exceeded normal values in 2/28 (92.9\%) patients with metastatic ovarian tumors originating from gastric cancer. A recent study by Feng et al (17) reported that CA125 was positive in $20 / 39(61.5 \%)$ of patients with metastatic tumors from gastric cancer; these authors also reported that the positive rates of other tumor markers were $18,38.5$ and $23.1 \%$ for carcinoembryonic antigen, CA19-9 and CA72-4, respectively, while $28.5 \%$ of the patients were negative for all markers. Thus, discriminating between primary and metastatic tumors is difficult, regardless of the use of these modalities.

The final diagnosis is made based on histopathological examination. Although the diagnosis of Krukenberg tumors depends on their characteristic light microscopic features with hematoxylin and eosin-stained sections, Krukenberg tumors often mimic other metastatic or primary ovarian tumors. The intracytoplasmic mucins in the signet ring cells are neutral and acidic (mostly sialomucins) and stain with Mayer mucicarmine, periodic acid-Schiff with diastase digestion and Alcian blue.

Immunohistochemically, adenocarcinoma cells are positive for cytokeratins (AE1/AE7) and epithelial membrane antigen, and negative for vimentin and inhibin (47). Immunohistochemical evaluation may also help distinguish primary ovarian from metastatic carcinomas. Furthermore, the cytokeratin $(\mathrm{CK}) 7 / \mathrm{CK} 20^{+}$immunophenotype favors primary ovarian carcinoma, whereas the $\mathrm{CK} 7 / \mathrm{CK} 20^{+}$or $\mathrm{CK} 7^{+} / \mathrm{CK} 20^{+}$immunophenotypes (CK20 positivity, in particular) favor metastatic gastrointestinal carcinoma $(47,48)$. Moreover, MUC5 AC suggests a metastatic gastric carcinoma over primary ovarian adenocarcinoma (48).

Regarding uterine metastases, women with abnormal uterine bleeding require histopathological evaluation. Histologically, metastatic carcinoma to the uterine corpus is characterized by focal occurrence, lack of necrosis and the lack of accompanying premalignant changes in the endometrium (49). As regards metastatic adenocarcinoma to the uterine cervix from gastric cancer, Imachi et al (43) reviewed 16 patients and reported that $9 / 16(56.3 \%)$ patients exhibited abnormal cytological findings. However, 8/14 (57.1\%) of the patients showed no abnormal findings on colposcopy. Noncontiguous spread of hematological, lymphatic or Fallopian tube etiology usually results in diffuse uterine enlargement with complete loss of the junctional zone on MRI $(50,51)$. The uterus may exhibit high or low signal intensity on T2-weighted imaging, and there may be heterogeneous enhancement. However, in some cases, a discernible mass may not be identified on MRI $(30,50)$. Physicians should not hesitate to perform random biopsies from the uterine cervix and/or endometrial curettage when a secondary tumor from the stomach is suspected, even when cytology, colposcopy and/or MRI fail to show any findings suggestive of malignancy.

\section{Management and prognosis}

The prognosis of patients with gastric cancer with metastasis to the ovaries has been reported to be poorer compared with that of other primary tumors $(3,14,23)$. Surgical intervention for these patients consists mainly of palliative resection to relieve the symptoms associated with a sizeable pelvic mass (19). 
Surgical tumor debulking followed by platinum-based chemotherapy is the standard therapeutic strategy for primary ovarian cancers. Kim et al (52) reported that the absence of residual disease after treatment and a limited disease extent favorably affected the prognosis of patients with metachronous Krukenberg tumors from gastric cancer. Feng et al (17) reported that metastatic peritoneal seeding was the determining factor affecting the prognosis of gastric cancer patients with ovarian metastasis. These results suggest that surgical resection without gross residual disease may improve the prognosis of patients with ovarian metastasis from gastric cancer. However, the number of related studies is limited, and some studies have reported conflicting results. Kobayashi et al (22) reported that the median survival following detection of the ovarian tumor was significantly longer in patients who underwent curative resection compared with those who received noncurative resection (30.4 vs. 10.3 months, respectively). Cheong et al (19) reported that complete resection of gross metastases from gastric cancer significantly improved the overall survival (18 vs. 9 months) and the progression-free survival ( 8 vs. 5 months). Jun et al (53) also reported that the survival of patients undergoing tumor resection without residual tumor was significantly longer compared with that of patients who underwent only palliative resection (23.7 vs. 6.0 months, respectively). By contrast, Ayhan et al (23) reported that optimal cytoreductive surgery significantly prolonged the median survival in patients with ovarian metastasis from colorectal cancer, but not in those with metastasis from gastric cancer. Kim et al (20) also suggested that there is a survival benefit from aggressive surgery in patients with colorectal cancer, but not in those with gastric cancer. However, these studies were based on a retrospective study including a small number of subjects. Prospective randomized trials are required to fully elucidate the significance of cytoreductive surgery in patients with ovarian metastasis from gastric cancer.

\section{Conclusions}

The prognosis of patients with gastric cancer with metastasis to the ovaries is poorer compared with that of other primary tumors. Although the results of cytoreductive surgery are encouraging, the optimal therapeutic strategies for such patients have not been fully established. A recent retrospective multicenter study in France by Brieu et al (1) evaluated the efficacy of modern chemotherapy regimens, such as platinum, irinotecan, taxane plus platinum, or epirubicin plus platinum, in the first-line treatment of patients with ovarian metastases from gastric cancer following oophorectomy. The investigators reported that the median overall survival of patients undergoing oophorectomy was significantly different compared with that of patients without oophorectomy (26.9 vs. 10.6 months, respectively), suggesting that surgical tumor debulking plus appropriate chemotherapy may improve the prognosis of patients with ovarian metastasis from gastric cancer.

\section{References}

1. Brieau B, Auzolle C, Pozet A, Tougeron D, Bouché O, Soibinet P, Coriat R, Prieux C, Lecomte T, Goujon G, et al: Efficacy of modern chemotherapy and prognostic factors in patients with ovarian metastases from gastric cancer: A retrospective AGEO multicentre study. Dig Liver Dis 48: 441-445, 2016.
2. Mazur MT, Hsueh S and Gersell DJ: Metastases to the female genital tract. Analysis of 325 cases. Cancer 53: 1978-1984, 1984.

3. Demopoulos RI, Touger L and Dubin N: Secondary ovarian carcinoma: A clinical and pathological evaluation. Int J Gynecol Pathol 6: 166-175, 1987.

4. Moore RG, Chung M, Granai CO, Gajewski W and Steinhoff MM: Incidence of metastasis to the ovaries from nongenital tract primary tumors. Gynecol Oncol 93: 87-91, 2004.

5. Skírnisdóttir I, Garmo H and Holmberg L: Non-genital tract metastases to the ovaries presented as ovarian tumors in Sweden 1990-2003: Occurrence, origin and survival compared to ovarian cancer. Gynecol Oncol 105: 166-171, 2007.

6. de Waal YR, Thomas CM, Oei AL, Sweep FC and Massuger LF: Secondary ovarian malignancies: Frequency, origin, and characteristics. Int J Gynecol Cancer 19: 1160-1165, 2009.

7. Fujiwara K, Ohishi Y, Koike H, Sawada S, Moriya T and Kohno I: Clinical implications of metastases to the ovary. Gynecol Oncol 59: 124-128, 1995.

8. Yada-Hashimoto N, Yamamoto T, Kamiura S, Seino H, Ohira H, Sawai K, Kimura T and Saji F: Metastatic ovarian tumors: A review of 64 cases. Gynecol Oncol 89: 314-317, 2003.

9. Turan T, Aykan B, Koc S, Boran N, Tulunay G, Karacay O, Erdogan Z and Kose F: Analysis of metastatic ovarian tumors from extragenital primary sites. Tumori 92: 491-495, 2006.

10. Kiyokawa T, Young RH and Scully RE: Krukenberg tumors of the ovary: A clinicopathologic analysis of 120 cases with emphasis on their variable pathologic manifestations. Am J Surg Pathol 30: 277-299, 2006.

11. Yamanishi Y, Koshiyama M, Ohnaka M, Ueda M, Ukita S, Hishikawa K, Nagura M, Kim T, Hirose M, Ozasa H, et al: Pathways of metastases from primary organs to the ovaries. Obstet Gynecol Int 2011: 612817, 2011.

12. Hale RW: Krukenberg tumor of the ovaries. A review of 81 records. Obstet Gynecol 32: 221-225, 1968.

13. Holtz F and Hart WR: Krukenberg tumors of the ovary: A clinicopathologic analysis of 27 cases. Cancer 50: 2438-2447, 1982.

14. Petru E, Pickel H, Heydarfadai M, Lahousen M, Haas J, Schaider $\mathrm{H}$ and Tamussino K: Nongenital cancers metastatic to the ovary. Gynecol Oncol 44: 83-86, 1992.

15. Li W, Wang H, Wang J, L v F, Zhu X and Wang Z: Ovarian metastases resection from extragenital primary sites: Outcome and prognostic factor analysis of 147 patients. BMC Cancer 12: 278, 2012 .

16. Kim NK, Kim HK, Park BJ, Kim MS, Kim YI, Heo DS and Bang YJ: Risk factors for ovarian metastasis following curative resection of gastric adenocarcinoma. Cancer 85: 1490-1499, 1999.

17. Feng Q, Pei W, Zheng ZX, Bi JJ and Yuan XH: Clinicopathologic characteristics and prognostic factors of 63 gastric cancer patients with metachronous ovarian metastasis. Cancer Biol Med 10: 86-91, 2013.

18. Akhan SE, Kilic G, Salihoglu Y, Bengisu E and Berkman S: Nongenital metastatic cancers of the ovary: A clinical analysis. Eur J Gynaecol Oncol 22: 379-383, 2001.

19. Cheong JH, Hyung WJ, Chen J, Kim J, Choi SH and Noh SH: Surgical management and outcome of metachronous Krukenberg tumors from gastric cancer. J Surg Oncol 87: 39-45, 2004.

20. Kim WY, Kim TJ, Kim SE, Lee JW, Lee JH, Kim BG and Bae DS: The role of cytoreductive surgery for non-genital tract metastatic tumors to the ovaries. Eur J Obstet Gynecol Reprod Biol 149: 97-101, 2010.

21. Ulbright TM, Roth LM and Stehman FB: Secondary ovarian neoplasia. A clinicopathologic study of 35 cases. Cancer 53: 1164-1174, 1984.

22. Kobayashi O, Sugiyama Y, Cho H, Tsuburaya A, Sairenji M, Motohashi $\mathrm{H}$ and Yoshikawa T: Clinical and pathological study of gastric cancer with ovarian metastasis. Int J Clin Oncol 8: 67-71, 2003.

23. Ayhan A, Guvenal T, Salman MC, Ozyuncu O, Sakinci M and Basaran M: The role of cytoreductive surgery in nongenital cancers metastatic to the ovaries. Gynecol Oncol 98: 235-241, 2005.

24. Miller BE, Pittman B, Wan JY and Fleming M: Colon cancer with metastasis to the ovary at time of initial diagnosis. Gynecol Oncol 66: 368-371, 1997.

25. Roma AA: Metastatic gastric adenocarcinoma primarily presenting in the fallopian tube. Ann Diagn Pathol 16: 63-66, 2012.

26. Stewart CJ, Leung YC and Whitehouse A: Fallopian tube metastases of non-gynaecological origin: A series of 20 cases emphasizing patterns of involvement including intra-epithelial spread. Histopathology 60: E106-E114, 2012. 
27. Rabban JT, Vohra P and Zaloudek CJ: Nongynecologic metastases to fallopian tube mucosa: A potential mimic of tubal high-grade serous carcinoma and benign tubal mucinous metaplasia or nonmucinous hyperplasia. Am J Surg Pathol 39: 35-51, 2015.

28. Piura B, Yanai-Inbar I, Rabinovich A, Zalmanov S and Goldstein J: Abnormal uterine bleeding as a presenting sign of metastases to the uterine corpus, cervix and vagina in a breas cancer patient on tamoxifen therapy. Eur J Obstet Gynecol Reprod Biol 83: 57-61, 1999.

29. Tsoi D, Buck M, Hammond I and White J: Gastric adenocarcinoma presenting as uterine metastasis-a case report. Gynecol Oncol 97: 932-934, 2005

30. Matsushita H, Fukase M, Takayanagi T and Ikarashi H: Metastatic gastric cancer mimicking an advanced cervical cancer: A case report. Eur J Gynaecol Oncol 32: 199-200, 2011.

31. Kumar NB and Hart WR: Metastases to the uterine corpus from extragenital cancers. A clinicopathologic study of 63 cases. Cancer 50: 2163-2169, 1982.

32. Stemmermann GN: Extrapelvic carcinoma metastatic to the uterus. Am J Obstet Gynecol 82: 1261-1266, 1961.

33. Martínez-Román S, Frumovitz M, Deavers MT and Ramirez PT: Metastatic carcinoma of the gallbladder mimicking an advanced cervical carcinoma. Gynecol Oncol 97: 942-945, 2005.

34. Lemoine NR and Hall PA: Epithelial tumors metastatic to the uterine cervix. A study of 33 cases and review of the literature. Cancer 57: 2002-2005, 1986.

35. Allard JE, McBroom JW,Zahn CM, McLeod D and Maxwell GL: Vaginal metastasis and thrombocytopenia from renal cell carcinoma. Gynecol Oncol 92: 970-973, 2004.

36. Mendese GW, Ayvazian PJ and Li C: Renal cell carcinoma presenting as a perineal mass: Case report and review of the literature. Urology 67: 847.e1-847.e2, 2006.

37. Yamamoto T, Mori T, Matsushima H, Sawada M and Kitawaki J: Late, isolated metastasis from poorly differentiated gastric cancer to the uterine cervix. Gynecol Oncol Case Rep 8: 17-20, 2014.

38. D'Arco F, Pizzuti LM, Romano F, Natella V, Laccetti E, Storto G Maurea S and Mainenti PP: MRI findings of a remote and isolated vaginal metastasis revealing an adenocarcinoma of the mid-sigmoid colon. Pol J Radiol 79: 33-35, 2014.

39. Dehner LP: Metastatic and secondary tumors of the vulva. Obstet Gynecol 42: 47-57, 1973.

40. Neto AG, Deavers MT, Silva EG and Malpica A: Metastatic tumors of the vulva: A clinicopathologic study of 66 cases. Am J Surg Pathol 27: 799-804, 2003.
41. Koyama T, Mikami Y, Saga T, Tamai K and Togashi K: Secondary ovarian tumors: Spectrum of CT and MR features with pathologic correlation. Abdom Imaging 32: 784-795, 2007.

42. Outwater EK, Wagner BJ, Mannion C, McLarney JK and Kim B: Sex cord-stromal and steroid cell tumors of the ovary. Radiographics 18: 1523-1546, 1998.

43. Imachi M, Tsukamoto N, Amagase H, Shigematsu T, Amada S and Nakano H: Metastatic adenocarcinoma to the uterine cervix from gastric cancer. A clinicopathologic analysis of 16 cases. Cancer 71: 3472-3477, 1993.

44. Treszezamsky A, Altuna S, Díaz L, Vighi S and Sardi J: Metastases to the uterine cervix from a gastric carcinoma presenting with obstructive renal failure: A case report. Int J Gynecol Cancer 13: 555-557, 2003.

45. Webb MJ, Malkasian GD Jr and Jorgensen EO: Factors influencing ovarian cancer survival after chemotherapy. Obstet Gynecol 44: 564-570, 1974.

46. Willmott F, Allouni KA and Rockall A: Radiological manifestations of metastasis to the ovary. J Clin Pathol 65: 585-590, 2012.

47. Al-Agha OM and Nicastri AD: An in-depth look at Krukenberg tumor: An overview. Arch Pathol Lab Med 130: 1725-1730, 2006.

48. Fazzari C, Fedele F, Pizzi G, Crisafulli C, Parisi A and Caruso RA: Krukenberg tumour of the ovary: A case report with light microscopy, immunohistochemistry and electron microscopy study. Anticancer Res 28 (2B): 1417-1420, 2008.

49. Kumar A and Schneider V: Metastases to the uterus from extrapelvic primary tumors. Int J Gynecol Pathol 2: 134-140, 1983.

50. Metser U, Haider MA, Khalili K and Boerner S: MR imaging findings and patterns of spread in secondary tumor involvement of the uterine body and cervix. AJR Am J Roentgenol 180: 765-769, 2003

51. Sohaib SA, Verma H, Attygalle AD and Ind TE: Imaging of uterine malignancies. Semin Ultrasound CT MR 31: 377-387, 2010.

52. Kim HK, Heo DS, Bang YJ and Kim NK: Prognostic factors of Krukenberg's tumor. Gynecol Oncol 82: 105-109, 2001.

53. Jun SY and Park JK: Metachronous ovarian metastases following resection of the primary gastric cancer. J Gastric Cancer 11: $31-37,2011$ 